\title{
16
}

\section{Analysis and design of production and control structures}

\author{
M.J. Verweij and A.J.R. Zwegers \\ Department of Technology Management \\ Eindhoven University of Technology, Pav. U21 \\ P.O. Box 513, $5600 \mathrm{MB}$ Eindhoven, the Netherlands \\ Tel: +31-40-2472444 Fax: +31-40-2451275 \\ Email:mvw@tm.tue.nl
}

\begin{abstract}
The objective of this paper is to illustrate the relationship between production structure and control structure. Identified production basic types (PBTs) and control basic types (CBTs) are used, that allow one to quickly analyse existing structures, and to develop alternatives. Features such as product variety, and (variations in) production volume provide the link between individual PBTs and CBTs as well as the link between configurations of the basic types. (Configurations of the) production and control modules are redesigned in an integral approach. The basic types visualise the effects of applying different design strategies.
\end{abstract}

\section{Keywords}

Production Management, Production Redesign, Control Structure, Design Strategies.

\section{INTRODUCTION}

Enterprises face internal modifications to cope with severe competition in the global marketplace. In order to survive at the global market, efficient and flexible operation is essential. Therefore, enterprises are obliged to adapt their production systems and control systems according to the external demands. However, because the systems change frequently, discrepancies might occur between a production

Advances in Production Management Systems N. Okino, H. Tamura \& S. Fujii (Eds.) (C) 1998 IFIP. Published by Chapman \& Hall 
system and its accompanying control system. In course of time, the production system and the control system do not 'match' anymore.

The objective of this paper is to illustrate the relationship between production structure and control structure. We focus on companies that are characterised by small batch manufacturing. We use a number of identified production basic types and control basic types. The use of basic types allows one to quickly analyse existing structures, and to develop alternatives. In addition, we explain the effects of design strategies upon production and control structures. We take the design strategies as distinguished by Galbraith (1973) as starting point. In this paper, we argue that companies should take production, control (and information) aspects into account together, and not sequentially. The results are obtained by literature study, and by practical experiences with the basic types in industrial applications (Verweij, et al., 1995).

We have found some relations between production structure and control structure. For example, the most suitable control type for a manufacturing cell is the heterarchical structure. In addition, it appeared that the basic types provide good means to demonstrate the effects of some design strategies. The application of the design strategies in practice implies that both the production and the control structure will be affected. Changing from one production basic type to another mostly leads to changing the control basic type as well, and vice versa.

\section{PRODUCTION BASIC TYPES AND CONTROL BASIC TYPES}

\subsection{Production Basic Types}

The Production Description Language (PDL) is a tool to describe, analyse, and redesign production organisations. The PDL describes an organisation by analysing the structure of the organisation's production processes and supporting processes. These processes consist of a number of mutual dependent activities that are grouped into modules. A production organisation can be constructed by combining a number of production and supporting modules.

The PDL contains descriptions of a number of basic types for production modules: the Production Basic Types (PBTs). The PBTs provide an overview of the possibilities to structure the production processes. They are not meant to classify production structures but to typify them, to identify relevant dimensions for a design of a production structure, and to indicate extreme values for these dimensions. Figure 1 presents the six PBTs that have been elaborated (Verweij, et al., 1995; Verweij, 1995). 


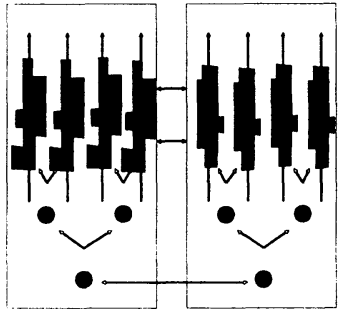

Functional departments

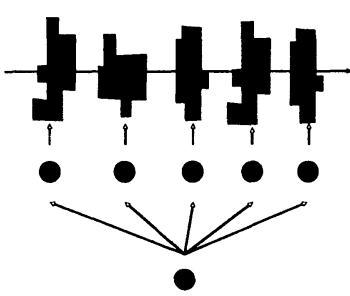

Multi-productline
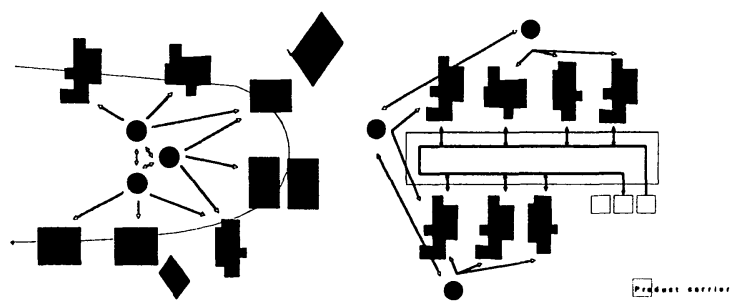

Manufacturing cell Flexible Manufacturing System

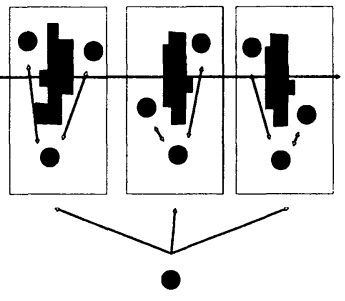

Flow dock

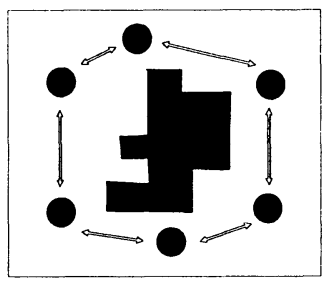

Dock

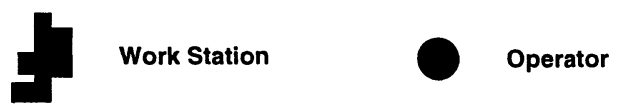

Figure 1 Production Basic Types

Each Basic Type has certain advantages and disadvantages that influence the performance in certain situations. The performance can be measured by the costs, the throughput times and the quality of the realisation of the required production programme. Different characteristics of the production programme such as product variety and complexity will lead to different choices of Basic Types or configurations of Basic Types. The PBTs can be mutually compared on their relative performance in relation to characteristics of the production programme. In this way, a suitability profile is created of the situations in which the Basic Types realise a better or worse performance. These suitability profiles have been presented in (Verweij, 1995).

\section{Functional department}

In a functional department, the work stations are connected in a parallel way, and they are not specialised. The products follow variable routings, only passing one of the work stations in the department. On each work station, one task is executed. Every work station is operated by one operator; possibilities exist to operate a few work stations at the same time. All supporting processes are separated from the production process. Raw materials, products and tools are distributed to the 
department from a central point. Quality assurance and process planning have been centralised as well. Finally, coordination takes place from a central point also.

\section{Manufacturing cell}

A manufacturing cell consists of a number of serially connected work stations. The products follow a fixed routing between the work stations. On every work station one task is executed; the work stations have been specialised for a specific group of products. Supporting processes such as storage, internal transport, quality assurance, tool management, and process planning are executed in the cell. Coordination between the work stations is executed in the cell as well.

\section{Flexible Manufacturing System (FMS)}

In a Flexible Manufacturing System (FMS), the work stations are serially connected by an automated materials handling system. This system also serves as a buffer between the work stations in which products may be stored. The routing of the products between the work stations is variable. Tool management has been automated as well. Process planning is executed outside the FMS in a central department. Coordination of the activities and quality assurance take place internally in the department.

\section{Multi-product line}

A multi-product line consists of a number of serially connected work stations with small buffers between them (or without buffers). The products follow a fixed routing between the work stations. On every work station, one task is executed. The output per time period is equal for every work station. The work stations are specialised for the required production programme. Storage of parts takes place in the department. The other supporting processes (process planning, quality assurance, transport of parts and finished products, tool management) are executed in other departments separated from the production. Coordination of the activities takes place centrally.

\section{Flow dock}

In a flow dock, a number of work stations are connected serially. The products follow a fixed routing. On each work station, a number of tasks are executed simultaneously on one product. The work stations are specialised for a certain group of products. The average output per time period of each work station has to be equal but the amount of work may vary by varying the number of parallel tasks. Storage, transport, tool management, process planning and quality assurance take place in the module. The flow dock is coordinated from a central point.

\section{Dock}

A dock consists of one integrated work station in which all activities are executed. These activities are coordinated in the dock. The dock is responsible for the output. 
Process planning takes place centrally. All other supporting processes take place in the module.

\subsection{Control Basic Types}

In addition to the Production Basic Types, three Control Basic Types (CBTs) have been identified. They are based on the work by Dilts, et al., (1991) on the evolution of control architectures and are represented in Figure 2. Note that unlike in (Dilts, et al., 1991), circles represent control entities, whereas production modules are portrayed as rectangles. Control entities might be information systems as well as human beings.

\section{Proper Hierarchical Control}

In a proper hierarchical control system, a specific controller dictates all activities of the subordinate (slave) level. The subordinates - whether they are production modules or lower level controllers - are not allowed to refuse the commands from the upper level controller. Control decisions are operated downwards, with status reporting operating upwards. Aggregate decisions are made at the highest levels. These decisions are filtered down and more detail is added as they pass through to the lower levels. Detail of information increases with each lower control level, whereas the time period for its consideration decreases.

A configuration of hierarchical controllers is characterised by a philosophy of 'control levels' and contains several control modules arranged in a pyramidal structure. Each level has its own purpose and function. At the top of the hierarchy is a single controller which is responsible for setting global goals and formulating long-range strategies that commit the entire hierarchical structure to coordinated actions which would result in achievement of the selected goals.

\section{Modified Hierarchical Control}

Just like in the previous Control Basic Type, in a modified hierarchical control system, controllers assume established supervisor/subordinate relationships with lower/upper level controllers. However, the main distinction between the two basic

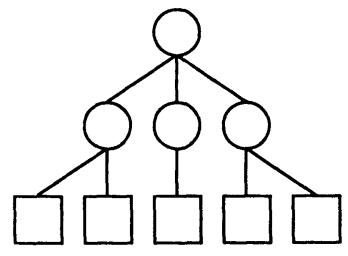
proper hierarchical
control

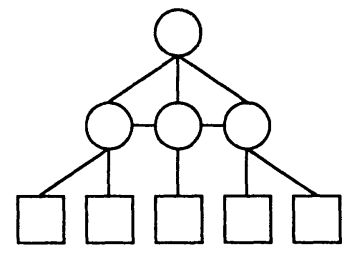

modified hlerarchical control

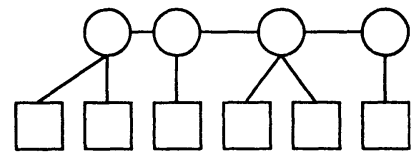

heterarchical control

Figure 2 Control Basic Types 
types lies in the degree of autonomy; modified hierarchical controllers are equipped with some amount of self-sufficiency (or autonomy) with respect to higher level controllers. This relative autonomy loosens the master/slave relationships between controllers; a controller acts as an intelligent assistant to the host and not as a slave. At the same hierarchical level, some degree of coordination among controllers is required to carry out a sequence of activities initiated by a command from a supervising controller.

\section{Heterarchical Control}

In a heterarchical control system, locally distributed, autonomous controllers communicate with each other without the master/slave relationship. Full local autonomy and a cooperative approach to decision making are the main features. Supervisory decision making is located locally at the point of information gathering rather than in a central location.

Cooperation between controllers is usually arranged via a negotiation procedure. The most important characteristic of a cooperative protocol is that it allows any module to refuse the transfer or acceptance of a message based on its own knowledge of its own status. These characteristics are necessary to ensure that full local autonomy is maintained during the cooperative process. By using a negotiation-based protocol for cooperation, controllers negotiate with each other to arrange operations such as scheduling and routing of work parts.

\section{Suitability of the Control Basic Types}

Like the PBTs, the CBTs also have certain advantages and disadvantages that influence their performance in certain situations. This performance depends on dimensions such as uncertainty, product variety, and production volume.

Galbraith (1973) defines uncertainty as the difference between the amount of information required to perform a task and the amount of information already possessed by the organisation. The amount of information needed to perform a task is dependent among others on the diversity of the outputs provided. Uncertainty occurs in the future demand concerning time, size and specifications of the products to be produced. In addition, the number of different input resources such as the required materials and capacity utilised plays a role. Finally, the amount of information required to perform a task depends on the level of goal difficulty or performance, as uncertainty might appear about the progress during execution of the production process (Rijn, 1985).

Heterarchical control systems, especially those equipped with negotiation capabilities, such as agent based systems, are claimed to be suitable for coping with uncertainty in product specifications, i.e. product variety. The self-organising way with which the manufacturing system structures itself should allow an unlimited routing flexibility between production modules. Evidently, routing flexibility is directly connected to the capacity of dealing with product variety. Thanks to the negotiation mechanism, and provided the production modules 
required for a certain product are actually present in the system, a part should always be able to 'find its way' through the plant in search of production machines able to manufacture it (Cantamessa, 1995).

Concerning production volumes, heterarchical control systems are usually introduced in view of large manufacturing systems and considerable production volumes. In systems with many machines working with high volumes made up of small batches, mathematical programming-based methods for production management suffer because of computational complexity. In such cases, the negotiation capabilities of heterarchical control systems might therefore have a good chance of proving themselves to be superior. On the other hand, with small scale production and large product variety, traditional, hierarchical scheduling methods are normally proposed; in these cases, the superiority of the heterarchical control systems should carefully be verified since there is no a priori evidence that it should prove to be superior (Cantamessa, 1995).

Hierarchical control systems are preferred when there is little uncertainty. If uncertainty is low, i.e. there are few fluctuations in product specifications and production volume, much of the operations can be preplanned. During the actual execution, little additional knowledge is acquired, and optimal schedules can be computed before actual operation. Then, hierarchical control systems should be favoured for an optimal coordination of production modules.

\section{RELATIONS BETWEEN PBTS AND CBTS}

A discussion on the relation between Production Basic Types and Control Basic Types affects two kinds of relationships: control employed within a production module, and control employed in a configuration of production modules, i.e. the coordination between production modules.

Discussing the control employed within a production module, it can be argued that there is a link between the choice for a control structure and for individual PBTs. The design of a control structure mainly depends on characteristics such as uncertainty, product variety, and (variations in) production volume. The choice for a specific PBT depends on characteristics such as quantity, variety, organisational and physical complexity, and fluctuations in product mix and production volume. So, features such as product variety, and (variations in) production volume provide the link between individual PBTs and CBTs. This link can be visualised by the combination of a Production Basic Type and a Control Basic Type. That is, inside a certain PBT a specific basic control type can be employed. The descriptions of the PBTs in section 2.1 illustrate that one CBT is more appropriate for a certain PBT than others. Table 1 depicts the most suitable Control Basic Types for the six Production Basic Types. 
Table 1 Relationships between Production Basic Types and Control Basic Types

\begin{tabular}{|c|c|c|c|c|c|c|}
\hline & $\begin{array}{l}\text { Func- } \\
\text { tional } \\
\text { Depart- } \\
\text { ment }\end{array}$ & $\begin{array}{l}\text { Manu- } \\
\text { facturing } \\
\text { Cell }\end{array}$ & $\begin{array}{l}\text { Flexible } \\
\text { Manu- } \\
\text { facturing } \\
\text { System }\end{array}$ & $\begin{array}{l}\text { Multi- } \\
\text { Product } \\
\text { Line }\end{array}$ & $\begin{array}{l}\text { Flow } \\
\text { Dock }\end{array}$ & Dock \\
\hline $\begin{array}{l}\text { Proper } \\
\text { Hierarchical } \\
\text { Control }\end{array}$ & & & & & & \\
\hline $\begin{array}{l}\text { Modified } \\
\text { Hierarchical } \\
\text { Control }\end{array}$ & & & & & & \\
\hline $\begin{array}{l}\text { Heterarchical } \\
\text { Control }\end{array}$ & & & & & & \\
\hline
\end{tabular}

Also decisions must be made upon the control and coordination between configurations of production modules. A configuration of production modules can consist of a number of production modules of the same PBTs, but might consist of a number of different PBTs as well. We argue that the relation between a configuration of PBTs and its control structure is not as strong as the link between a PBT and the internal PBT control structure. For example, the next section illustrates that, if internal changes in functional departments are made, causing it to transform into another PBT, its internal control structure will change as well. On the other hand, a configuration of functional departments might be controlled by a pyramid of hierarchical controllers in case the production volume is stable and can be planned well in advance. The same configuration of functional departments might be controlled by direct communication between the departments in case many capacity adaptations are necessary because of fluctuations in the production volume. Mutual dependency between the production modules might play an additional role in the choice of the most appropriate control structure in addition to the already mentioned characteristics such as uncertainty and product variety.

\section{DESIGN STRATEGIES AND THEIR CONSEQUENCES}

The best way to organise is contingent upon the uncertainty and diversity of the basic task being performed by an organisational unit (Galbraith, 1973). Following the changing external demands, enterprises are obliged to adapt their production systems and control systems.

Traditional design approaches follow a P-C-I approach in which design of the production processes $(\mathrm{P})$, the control modules $(\mathrm{C})$, and the information systems (I) 

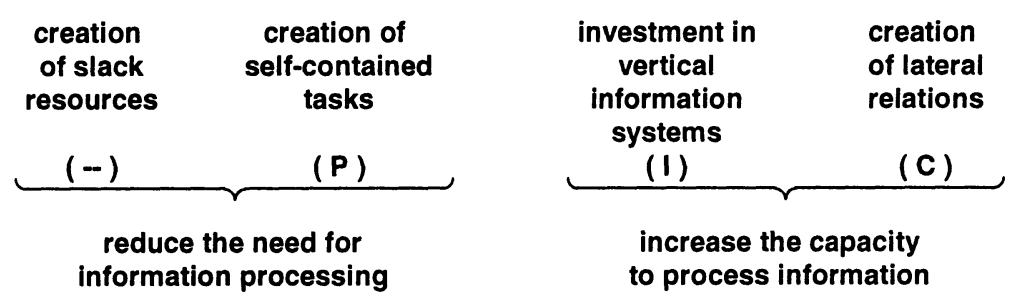

Figure 3 Galbraith's organisation design strategies

are handled in a sequential way. However, more and more the increasing frequency of system changes and the increased reciprocity of the three aspects $\mathrm{P}, \mathrm{C}$ and I require an integral design approach (Kusters, et al., 1995).

In addition to rules, hierarchical referral and goal setting, Galbraith has described four organisational design strategies (Galbraith, 1973). Figure 3 depicts these design strategies. Obviously, the design strategies focus upon different aspects; their main points of attention in a redesign process are shown between parentheses. For example, the creation of lateral relations increases the capacity to process information by adjusting the control structure, whereas the creation of selfcontained tasks interferes with the production structure. However, the developments described above require the other aspects to be redesigned and adjusted as well. The PBTs and CBTs can be used to depict these interferences between the various aspects while applying different design strategies. For each design strategy, this is illustrated by an example.

\subsection{Creation of Slack Resources}

An organisation can reduce the number of exceptions that occur by simply reducing the required level of performance. Slack resources are an additional cost to the organisation. It implies the establishment of additional production and/or control modules in the organisation. The basic structure, however, will not be changed. If, for instance, additional functional departments are created in the organisation, also additional control modules will have to be created that fit in the existing control structure.

\subsection{Creation of Self-contained Tasks}

The creation of self-contained tasks involves the change from the functional task design to one in which each group has all the resources it needs to perform its task. A production structure based on functional departments or multi-product lines will be changed into one based on manufacturing cells or Flexible Manufacturing Systems or into one based on flow docks or docks. Table 1 immediately depicts the 
consequences for the internal control structure: proper hierarchical control will have to be replaced by modified hierarchical control or heterarchical control.

\subsection{Investment in Vertical Information Systems}

Investments in vertical information systems, such as management information systems or MRP systems, mainly focus on the information processes in the organisation. These kinds of investments will increase the capacity of the decision maker. The information processes are not the prime focus of this paper; no Information Basic Types have been defined, although this might be possible. In general, it can be said that investments in vertical information systems will tend an organisation to create more hierarchical control structures which might evolve to PBTs like functional departments and multi-product lines.

\subsection{Creation of Lateral Relations}

The employment of lateral decision processes moves the level of decision making down to where the information exists rather than bringing it up to the points of decisions. Though mainly influencing the control structure, this solution also provides new possibilities to further adapt the production structure. Take a certain production module that is characterised as a multi-product line with hierarchical control as an example. When the task uncertainty increases, the organisation might employ the creation of lateral relations. This way, the control type of the module changes to the modified hierarchical or heterarchical type. Then, it is a small step to re-organise the line into a manufacturing cell.

\subsection{Designing the production and control structure}

The design strategies described by Galbraith either involve the creation of additional capacity or slack in the existing organisation or a transition to a new organisation. Each design strategy for such a transition has its prime focus on a certain aspect, whereas the relationships between PBTs and CBTs indicate the interferences with the other aspects to be redesigned in an integral way. The overview of transitions between PBTs depicted in Figure 4 might support the execution of the described design strategies. The arrows in the picture indicate the most common transitions from one production basic type to another. Note that a thick arrow indicates a more frequent transformation than a thin arrow. 


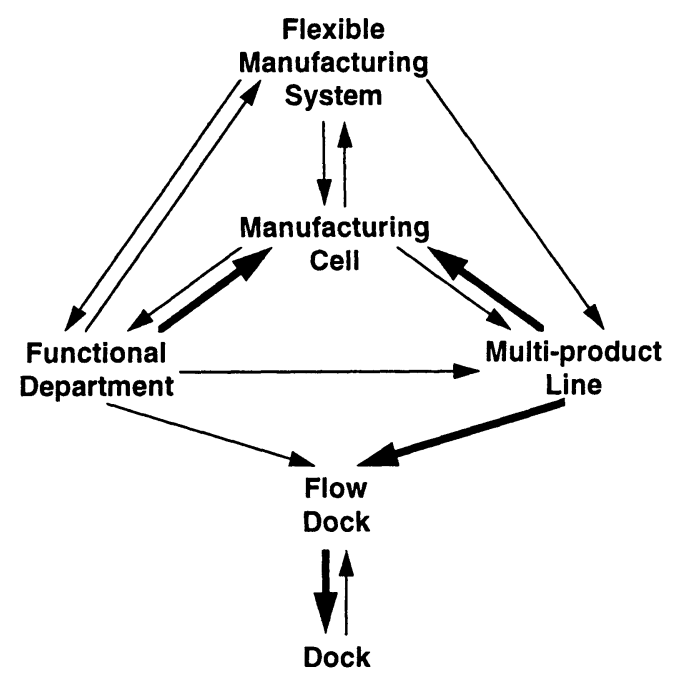

Figure 4 Transitions between PBTs

Using Table 1, the additional changes in the control structure can be derived as well. Production Basic Types on the left and right side will be controlled hierarchically, while in the middle PBTs more distributed control types are used. Starting from an existing structure, possible alternatives can be developed and analysed. For instance, a production structure consisting of functional departments most frequently is transformed to a structure consisting of manufacturing cells. Other possibilities are the use of flexible manufacturing systems, multi-product lines or flow docks. The proper hierarchical control structure mostly used in the functional departments will change into a modified hierarchical or heterarchical control structure. In addition to a changed control structure within the PBTs, also the control of the newly built configuration of PBTs/CBTs might be changed due to the considerations described in section 3. Redesign of the (configuration of the) production and control modules are executed in an integral approach. However, in this paper we do not elaborate this aspect further.

\section{CONCLUSIONS}

To survive at the global market, efficient and flexible operation is essential. Following the changing external demands, enterprises are obliged to adapt their production and control structure almost continuously. More and more, integral design approaches (taking into account production, control and information aspects in a more interactive way) are required to achieve this.

Production basic types and control basic types have been used to illustrate the relationships between the production structure and the control structure in an 
organisation. Also the consequences of various design strategies have been discussed. The basic types and their mutual relationships provide the possibility to quickly develop alternative redesigns to analyse changes in both the production and the control structure so that a well-based decision on a new organisation concept can be taken.

\section{REFERENCES}

Cantamessa, M. (1995). A few notes upon Agent-based Modelling of Manufacturing Systems, in Proceedings of the CIM at Work conference (ed. J.C. Wortmann), pp. 301-317.

Dilts, D.M., N.P. Boyd, and H.H. Whorms. (1991). The evolution of control architectures for automated manufacturing systems. Journal of Manufacturing Systems, 10(1), pp. 79-93.

Galbraith, J. (1973). Designing Complex Organizations. Addison-Wesley, Reading, Massachusetts.

Kusters, R.J., F. van Eijnatten, and H. van Ooijen. (1995). Business Process Redesign: directions for new research (in Dutch), in Proceedings of NOBO '95, pp. 13-20.

Rijn, Th.M.J. (1985). Producing by informing - Information requirements for various production situations (in Dutch). Kluwer.

Verweij, M.J., M.A. Rotzoll, and A. Müller. (1995). Redesign of small batch manufacturing: Basic Type Modelling, in Proceedings of CIRP '95, pp. 337345.

Verweij, M.J. (1995). Describing, Analysing and Designing with the Production Description Language. Report EUT/BDK/74. Eindhoven University of Technology.

\section{BIOGRAPHY}

Maurits Verweij received his M.Sc. degree from the Eindhoven University of Technology in 1991. Currently, he is preparing his $\mathrm{Ph} . \mathrm{D}$. thesis at the same university and he is employed as a management consultant by Berenschot B.V. in Utrecht, the Netherlands. Both his research and consultant activities focus on production management and redesign.

Arian Zwegers received his M.Sc. degree from the Eindhoven University of Technology in 1993. Currently, he is preparing his Ph.D. thesis at the same university. For two years, he was involved in the ESPRIT project VOICE II. His research interests include shop floor control architectures, enterprise reference architectures, and software architecting. 